\title{
ANORECTAL MALFORMATION FEMALE CHILDREN ONE STAGE PROCEDURE ASARP
}

\author{
Dhirendra Shrivastava ${ }^{1}$
}

${ }^{1}$ Assistant Professor, Department of Paediatrics Surgery, Gandhi Medical College, Bhopal.

ABSTRACT
CONTEXT
Anorectal malformation is a common congenital defect in children and its management has evolved over the years. Anterior
sagittal approach is established as a popular technique for the correction of anovestibular fistula in children with anorectal
malformations due to advancement of technology magnification, muscle stimulation and precise placement of the anal canal within
the external sphincter complex.

\section{AIMS}

To study the outcome of anterior sagittal approach as single stage procedure for anorectal malformations in female children at tertiary care centre.

\section{SETTINGS AND DESIGN}

Paediatric Surgery Department, Gandhi Medical College, Bhopal. A retrospective analysis.

\section{METHODS}

24 female patients with congenital anorectal malformation who underwent Anterior Sagittal Anorectoplasty (ASARP) as a single stage procedure were reviewed. The surgical procedure and its outcome were evaluated.

\section{RESULTS}

There was no case that had significant haemorrhage during or after procedure; 4 cases had rent in the posterior vaginal wall which was managed adequately due to early detection. There were six cases with wound infection. One case had dehiscence; three cases developed constipation, which was managed with dilation and bowel training.

\section{CONCLUSION}

ASARP as a single stage procedure without colostomy cover should be considered as a preferable option for the management of anovestibular fistula in female children.

\section{KEYWORDS}

Anorectal Malformation, Anterior Sagittal Anorectoplasty, Anovestibular Fistula.

HOW TO CITE THIS ARTICLE: Shrivastava D. Anorectal malformation female children one stage procedure ASARP. J. Evolution Med. Dent. Sci. 2016;5(25):1339-1341, DOI: 10.14260/jemds/2016/315

\section{INTRODUCTION}

Anorectal malformation is a common cause of intestinal obstruction in children. The incidence is 1:4000-5000 live births worldwide, could vary in Indian patients. $(1,2)$ Vestibular fistula is the commonest Anorectal Malformation (ARM) in the female children. The classification and management of this condition has evolved over the years. The contribution by Stephen, Rehbein had evolved the surgical management of patients with anorectal malformation.(3-5) Different surgical techniques have been described for the correction of such anomalies including cutback procedure, anal transplantation, YV plasty and Posterior Sagittal Anorectoplasty (PSARP). PSARP was introduced by Alberto Pena and Devries in early 1980s and was used to correct ARM. $(3,4)$

Financial or Other, Competing Interest: None

Submission 12-02-2016, Peer Review 09-03-2016,

Acceptance 14-03-2016, Published 28-03-2016.

Corresponding Author:

Dr. Dhirendra Shrivastava,

Assistant Professor

Department of Paediatric Surgery,

Gandhi Medical College,

Bhopal.

E-mail: shridhirendra@gmail.com

DOI: $10.14260 /$ jemds $/ 2016 / 315$
In 1992, Okada introduced Anterior Sagittal Anorectoplasty (ASARP) for treatment of rectovestibular and anovestibular fistula in children with ARM. $(6,7)$ This can be done as staged procedure with covering colostomy or as single stage procedure.

This study was planned to analyse the results of ASARP as single stage procedure in management of anovestibular fistula in female children.

\section{METHODS}

Patients attending the Department of Paediatric Surgery between Jan 2008 and 2014 with the diagnosis of ARM with vestibular fistula were operated with ASARP procedure; 24 female patients of age between 1 month and 5 years were included in the study; 3 patients had previous cut back. In all the patients, enema washout was used to prepare the gut. In immediate postoperative period, patients were kept nil orally and on intravenous alimentation. Initial dressing was removed on second day and the operative site cleaned and povidone iodine ointment applied and later anal dilation was done.

Surgical Technique: Patient was positioned in a supine position with elevation of lower back to ensure good access to the perineal area and legs hanging from above in squatting position. The center of the external anal sphincter was assured 
by the anal dimple and electrical stimulation and marked with silk sutures and marking pen. A circumferential incision in the mucocutaneous junction was made around the ectopic anal orifice stay sutures silk 4-0 were taken to secure the anal manipulation during surgery and then a midline vertical skin incision was extended to reach up to the posterior limit of the external sphincter by staying in midline. The stay sutures to secure the vaginal wall were taken. The rectal wall was then meticulously dissected using dilator to guide the direction and plane from the posterior vaginal wall anteriorly with sharp and blunt dissection. Mobilization of the rectal pouch was continued laterally and posteriorly until it was free from attachments and reached the new anal site without tension. Perineal body reconstruction was started from above downwards with $3 / 0$ vicryl interrupted stitches, the rectal wall was included in 2 stitches to prevent retraction of the rectum. Sphincter complex was reconstructed.

Then the anoplasty was done with four stitches at 12, 3, 6,9 'o clock and two more in between them to create neo-anal opening with the Hegar dilator and the guide to ensure adequate sizing. The child was kept nil orally and on IV fluids for 5 days along with IV antibiotics and IV metronidazole. The wound was dressed with sterilized gauze piece for 24 hours and left open after that. One patient required covering pelvic colostomy, as she developed complete wound dehiscence in postoperative period. Once the wound healing was achieved, the patients were put on regular dilations.

\section{RESULTS}

The patients included in the study were in the age ranging from 1 month to 3.5 yrs. Associated anomalies were present in 32\% of patients, of which $45 \%$ were urological anomalies.

During the procedure three patients had vaginal wall tear, which was recognized early due to the supine position of the patients and was prevented from extending upwards. No significant haemorrhage was observed. Transfusion was given in four patients with anaemia (Table 1). The follow-up ranged between 7 months and 21 months. The external appearance of the perineum was satisfactory in all patients except three, who had wound infection and one dehiscence (Table 2).

Patient's manoeuvring during dissection was uneventful and subjectively better in all 24 cases of ASARP.

\section{DISCUSSION}

The 24 patients of this series with the age range of 1 month to 3.5 yrs. had satisfactory outcome in terms of cosmetic and functional correction with three patients having wound infection and dehiscence in one, although there was no incontinence in this series but three patients had constipation which was managed with dilatation, diet regulation and bowel training.

The classification of ARM is important in order to properly group the malformations with a view to improve its management. The embryological basis of the pathology has been extensively reviewed and has helped in the breakthroughs in the management of the disease.

Several surgical techniques have been described for the correction of anovestibular fistula, which usually results in an anal opening made in the center of the sphincter muscle complex. There is changing trend in the management of anorectal malformation with rectovestibular fistula as more patients with this condition are managed without a preliminary colostomy with good outcome. This is different from where an initial colostomy was done before the definitive procedure proper bowel preparation, before definitive surgery was adequate for those who had definitive surgery without a colostomy. One stage treatment of vestibular fistula has also been advocated by various other surgeons.(6,7) The advantage of this approach is that it avoids a colostomy and thus reducing the number of operations, although in some cases perineal infections and dehiscence may cause fibrosis.

While PSARP by Alberto Peña and Devries is popular since reported in $1980 .(3,5)$ but the prone position is not a convenient and comfortable for dissecting the plane between rectum and vagina, which lies more anteriorly. ASARP was introduced by Okada in 1992 for treatment of rectovestibular and anovestibular fistula.(6,7) The advantages of ASARP over the previously mentioned techniques are: Separation of posterior vaginal wall from rectum which is considered the most important step of the operation, surgery under direct vision the rectum is placed and anchored within the muscle complex and the perineal body is accurately reconstructed. There is also comfortable position of the patient and operative surgeon with good anatomical orientation during procedure. We did all our cases without covering colostomy. Some of the authors (Wakhlu et al.), advocated that operation on patients with rectovestibular fistula is safer under cover of a protective colostomy. $(8,9)$

Various surgeons have reported that there is no significant difference in multistage and single stage procedure.(10-17) Our series is small for statistical analysis, but the results are in accordance with these reported series outcome in terms of continence can be achieved by careful anterior sagittal surgical dissection. Anovestibular fistula can be repaired in one stage with meticulous pre- and postoperative bowel management. It is a good option in cases which are not able to afford prolonged hospitalization or are not willing for a colostomy.(13,14) We admitted all patients 48 hours prior to operation for bowel preparation and 5 days after operation to keep baby nil orally to avoid any bowel movement.

The ASARP procedure is recommended even for the management of perineal trauma in female children.(8) and as a redo operation for imperforate anus in properly selected patients with poor anorectal function following the primary operation with satisfactory results.(7) In a modification of the operation, the authors from Australia described the Neutral Sagittal Anorectoplasty (NSARP), which preserves a perineal skin bridge between the neo-anus and the posterior fourchette and the lavatory muscle for aesthetic appearance of the perineum.(18) Total gut irrigation preoperatively for 2 days and keeping the child nil orally for the first 5 postoperative days was our protocol to prevent contamination of the wound or to keep it to the minimum in the first postoperative week. Similar practice with excellent results was observed by Menon and Rao from Chandigarh, India, in the management of vestibular fistula by primary posterior sagittal anorectoplasty.(19) In the anterior approach (ASARP), the incision is much smaller and requires the division of only anterior fibers of external sphincter complex, while posteriorly it remains intact. Thus the amount of tissue dissection in posterior approach is more, therefore a larger area would be at risk in case if infection occurs. In the anterior approach anterior dissection, i.e. separation of vagina and rectum which is considered to be the 
most important part of the operation, takes place under direct vision, while in posterior approach this dissection is blind. In female ARMs, we have two basic aims at operation, viz. adequate separation of vagina from the rectum and adequate downward mobilization of rectum to perform a tension-free anastomosis with skin. In conclusion, there has been a significant change in the management of anorectal malformation with improved outcome. This has been due to better understanding of the embryological basis, pathological condition and refinements in the surgical treatment. The supine approach during surgery, better exposure, use of magnification and muscle stimulator for operative actions in ASARP makes it an important option for management of anovestibular fistula. The conventional vestibular fistulas can benefit from ASARP with or without colostomy cover depending on the preference and experience of the surgeon, ASARP with lithotomy can be preferred. It is suggested to do with colostomy cover during the initial period, but as soon as one is comfortable with it one can start performing without colostomy cover. This approach is recommended as an optimal technique for treatment of vestibular anus in girls by experienced surgeons with large series of cases and our results agrees with the published literature.

\begin{tabular}{|c|c|}
\hline Complications & Number of Patients \\
\hline Haemorrhage & 0 \\
\hline Vaginal wall tear & 2 \\
\hline Rectal wall injury & 0 \\
\hline Wound infection & 3 \\
\hline Wound dehiscence & 1 \\
\hline \multicolumn{2}{|c|}{ Table 1: Complications during ASARP } \\
\hline
\end{tabular}

\begin{tabular}{|c|c|}
\hline \multicolumn{2}{|c|}{ Followup } \\
\hline Anal stenosis & 1 \\
\hline Anal stricture & 0 \\
\hline Mucosal prolapse & 0 \\
\hline Constipation & 3 \\
\hline Incontinence & 0 \\
\hline Table 2: Followup after ASARP \\
\hline
\end{tabular}

\section{REFERENCES}

1. Stephens FD, Smith ED. Classification, identification and assessment of treatment of anorectal anomalies. Pediatr Surg Int 1986;1:200-5.

2. Stephen FD, Smith ED. Anatomy and function of the normal rectum and anus; individual deformities in the male; operative management of anal deformities. Anorectal malformations in children, Chicago, IL, Year Book 1971;212-73.

3. Pena A, Devries PA. Posterior sagittal anorectoplasty: important technical consideration and new applications. J Pediatr Surg 1982;17:796-811.
4. Pena A, Hong A. Advances in the management of anorectal malformation. Am J Surg 2000;108:370-6.

5. Holschneider AM, Jesch NK, Stragholz E, et al. Surgical methods for anorectal malformation from rehbein to pena: critical assessment of score systems and proposal for a new classification. Eur J Pediatr Surg 2002;12:7382.

6. Okada A, Kamata S, Imura K, et al. Anterior sagittal anorectoplasty for rectovestibular and anovestibular fistula. J Pediatr Surg 1992;27:85-8.

7. Okada A, Tamada H, Tsuji $\mathrm{H}$, et al. Anterior sagittal anorectoplasty as a redo operation for imperforate anus. J Pediatr Surg 1993;28:933-8.

8. Wakhlu A, Pandey A, Prasad A, et al. Anterior sagittal anorectoplasty for anorectal malformations and perineal trauma in the female child. J Pediatr Surg 1996;31:123640.

9. Wakhlu A, Kureel SN, Tandon RK, et al. Long-term results of anterior sagittal anorectoplasty for the treatment of vestibular fistula. J Pediatr Surg 2009;44:1913-9.

10. Shehata SM. Prospective long-term functional and cosmetic results of ASARP versus PASRP in treatment of intermediate anorectal malformations in girls. Pediatr Surg Int 2009;25:863-8.

11. Zamir N, Mirza FM, Akhtar J, et al. Anterior sagittal approach for anorectal malformations in female children: early results. J Coll Physicians Surg Pak 2008;18:763-7.

12. Kumar B, Kandpal DK, Sharma SB, et al. Single-stage repair of vestibular and perineal fistulae without colostomy. J Pediatr Surg 2008;43:1848-52.

13. Kulshrestha S, Kulshrestha M, Singh B, et al. Anterior sagittal anorectoplasty for anovestibular fistula. Pediatr Surg Int 2007;23:1191-7.

14. Aziz MA, Banu T, Prasad R, et al. Primary anterior sagittal anorectoplasty for rectovestibular fistula. Asian J Surg 2006;29:22-4

15. Gangopadhyay AN, Gopal SC, Sharma S, et al. Management of anorectal malformation in Varanasi India: a long term review single and 3 staged procedures. Pediatr Surg Int 2006;22:169-72.

16. Lui G, Yuan J, Geng J, et al. Treatment of high and intermediate anorectal malformation: one stage or 3 stages. J Pediatr Surg 2004;39:1466-71.

17. Uba AF, Chirdan LB, Ardill W, et al. Anorectal anomalies: a review of 82 cases seen at JUTH, Nigeria. Niger Postgrad Med J 2006;13:61-5.

18. Dave S, Shi EC. Perineal skin bridge and levator muscle preservation in neutral sagittal anorectoplasty (NSARP) for vestibular fistula. Pediatr Surg Int 2005;21:711-4.

19. Menon P, Rao KL. Primary anorectoplasty in females with common anorectal malformations without colostomy. J Pediatr Surg 2007;42:1103-6. 